\title{
Theoretical Conformational Analysis on Elastin Model Tetrapeptide Ac-Val-Pro-Gly-Gly-NHMe
}

\author{
Masahito OKA, ${ }^{*,+}$ Yoshihiro BABA, ${ }^{*}$ Akihiro KAGEMoto, ${ }^{*}$ \\ and Akio NAKAJIMA** \\ * Departments of General Education and ** Applied Chemistry, \\ Osaka Institute of Technology, \\ Omiya, Asahi-ku, Osaka 535, Japan
}

(Received January 19, 1989)

\begin{abstract}
To investigate the short-range interactions in elastin, conformational energy calculations using ECEPP were carried out for elastin model tetrapeptide, Ac-Val-Pro-Gly-GlyNHMe. The DCC* conformation is the most favorable one for Val-Pro-Gly portion in the model tetrapeptide. The bend in trans Ac-Val-Pro-Gly-Gly-NHMe are recognized at Pro-Gly and GlyGly portions with higher probability. Favorable bend type at Pro-Gly is type II and type V which is a distorted type II $\beta$-bend, and the summation of these bend probabilities is 0.60 which corresponds to the tendency of forming a type II $\beta$-bend at Pro-Gly supposed by experimental works in solution and crystal structure.

KEY WORDS Conformational Energy Calculation / ECEPP / Ac-ValPro-Gly-Gly-NHMe / Elastin / $\beta$-Bend /
\end{abstract}

Elastin is a protein which represents characteristic elasticities. Weis-Fogh and Anderson ${ }^{1}$ have shown that these characteristic behaviours are not caused by the normal rubber elasticity usually observed for cross-linked random-coiled polymers, but explained as energetical change caused by the change of backbone conformation and entropical change caused by structural change of immanent water molecules through the stretching of backbone conformation. These facts indicate that the origin of the elasticity of elastin exists in its amino acid sequences, and elastin molecule has several stable ordered structures whose stabilities depend on the external conditions.

In sequence studies of water soluble elastin, Gray et al..$^{2,3}$ recognized two distinct regions of elastin, Lys and Ala-rich region and Gly, Pro, and Val-rich region, and the latter region consists of three typical repeated sequences, Val-Pro-Gly-Gly, Val-Pro-Gly-Val-Gly, and Ala-Pro-Gly-Val-Gly-Val. They supposed that the characteristic elasticity of elastin would be concerned with these typical repeated sequences, and also proposed the "oiled coil" structure ${ }^{2}$ which is the loosed spiral containing $\beta$-bend conformation. In this model, Gly residues occupy the exterior positions exposed to solvent, while Pro, Val, and Ala residues are burried, and most of the amide protons and carbonyl oxygens were hydrated. While other models were proposed by Urry et $a l^{4,5}$ based on the experimental results, which are the temperature dependence of amide proton chemical shift, solventcomponent dependence of carbonyl chemical shift for several solvents and NOE (Nuclear Overhauser Enhancement) measurements in NMR, and CD measurements for model

\footnotetext{
* To whom all correspondence should be addressed.
} 
polypeptides, ${ }^{4}$ poly(Val-Pro-Gly-Gly), poly(Val-Pro-Gly-Val-Gly), and poly(Ala-ProGly-Val-Gly-Val), and model peptides. ${ }^{6,7}$ The basic structure of Urry's model is $\beta$-bend conformation at Pro-Gly, while that of Gray's model is $\beta$-bend conformation at Gly-Gly.

In this paper, as the first step of the conformational analysis of elastin model polypeptide, poly(Val-Pro-Gly-Gly), conformational energies of Ac-Val-Pro-Gly-Gly-NHMe were calculated with the conformational energy functions of ECEPP (Empirical Conformational Energy Program for Peptides) ${ }^{8}$ Stable conformations of Val-Pro-Gly-Gly sequence of poly(Val-Pro-Gly-Gly) are also analyzed theoretically considering only shortrange interactions. Conformational analysis of poly(Val-Pro-Gly-Gly) considering longrange interactions will be followed (manuscript in preparation).

\section{THEORETICAL}

The nomenclature and conventions adopted are those recomended by an IUPAC-IUB nomenclature commission. ${ }^{9}$ Conformational energy calculations of the Ac-Val-Pro-GlyGly-NHMe tetrapeptide were carried out with ECEPP. ${ }^{8}$

Minimization was carried out with the' Powell algorithm ${ }^{10}$ until the conformational energy did not change by more than 0.001 $\mathrm{kcal} \mathrm{mol}^{-1}$ between successive iterations. During minimization, all $\phi, \psi$, and $\chi$ for Val and Gly residues and $\psi$ for Pro residue were allowed to vary. All other backbone dihedral angles were fixed at $180^{\circ}$, except for cis peptide bond at Val-Pro $\left(\omega=0^{\circ}\right)$. All combinations of single-residue minima ${ }^{11}$ were used as starting conformations. The following conformations were also used as single-residue minima, i.e., $(\phi, \psi)=\left(-75^{\circ}, 140^{\circ}\right)$ and $\left(75^{\circ},-140^{\circ}\right)$ for Gly residue, $\psi=23^{\circ}$ for Pro. While $(\phi, \psi)=\left(-76^{\circ}\right.$, $\left.-26^{\circ}\right),\left(-87^{\circ},-26^{\circ}\right)$, and $\left(-142^{\circ}, 29^{\circ}\right)$ for Val residue were excluded because they situate in high energy regions for Val-Pro sequence.
The normalized Boltzmann factor $v_{i}$ is given by

$$
v_{i}=Q^{-1} \exp \left(-\Delta E_{i} / R T\right)
$$

where

$$
Q=\sum_{i=1}^{n} \exp \left(-\Delta E_{i} / R T\right)
$$

$\Delta E_{i}$ is the conformational energy difference of the $i$ th minimum from that of global minimum, $R T$ is the gas constant times the temperature, $n$ is the number of low-energy minima. The statistical average of the conformation-dependent quantity $A$ for the ensemble is defined by

$$
A=\sum_{i=1}^{n} A_{i} v_{i}
$$

A bend (occuring at $i+1$ and $i+2$ th residues) is defined as a conformation in which $R \leq 7 \AA$ ( $R$ is the distance between $i$ th $\mathrm{C}^{\alpha}$ and $i+3$ th $\mathrm{C}^{\alpha}$ atoms.) and also classified into eleven types given in Table I of ref 12. A polar hydrogen atom and an oxygen or nitrogen atom with an interatomic distance of less than $2.3 \AA$ are regarded to be hydrogen-bonded. Vicinal NH$\mathrm{C}^{\alpha} \mathrm{H}$ coupling constants ${ }^{3} J_{\mathrm{NH}-\mathrm{C}^{\alpha} \mathrm{H}}$ of Val residue for each conformation were computed with the following expression of Bystrov et al. ${ }^{13}$

$$
{ }^{3} J_{\mathrm{NH}-\mathrm{C}^{\alpha} \mathrm{H}}=9.4 \cos ^{2} \theta-1.1 \cos \theta+0.4
$$

where $\theta=\phi-60^{\circ}$, and those of Gly residue were computed with the following expression of Bystrov et al. ${ }^{13}$

$$
{ }^{3} J_{\mathrm{NH}-\mathrm{C}^{\alpha} \mathrm{H}}=-9.4 \cos ^{2} \theta-1.1 \cos \theta+14.9
$$

Conformational space is divided into 16 regions with the conformational letter codes shown in Figure 2 of ref 11.

\section{RESULTS}

There are 116 low-energy minima for AcVal-Pro-Gly-Gly-NHMe with trans Val-Pro peptide bond having $\Delta E<3 \mathrm{kcal} \mathrm{mol}^{-1}$, indicating that this peptide system is represented 
Table I. Calculated minimum energy conformations ${ }^{\mathrm{a}}$ of trans Ac-Val-Pro-Gly-Gly-NHMe

\begin{tabular}{|c|c|c|c|c|c|c|c|c|c|c|}
\hline \multirow{2}{*}{$\begin{array}{l}\text { Conformational } \\
\text { letter code }\end{array}$} & \multirow{2}{*}{$\frac{\Delta E^{\mathrm{b}}}{\mathrm{kcal} \mathrm{mol}}$} & \multirow{2}{*}{$\phi_{\mathrm{Val}}$} & \multirow{2}{*}{$\psi_{\mathrm{Val}}$} & \multirow{2}{*}{$\psi_{\mathrm{Pro}}$} & \multirow{2}{*}{$\phi_{\mathrm{Gly3}}$} & \multirow{2}{*}{$\psi_{\mathrm{G} 1 y 3}$} & \multirow{2}{*}{$\phi_{\mathrm{Gly} 4}$} & \multirow{2}{*}{$\psi_{\mathrm{Gly} 4}$} & \multicolumn{2}{|c|}{ Bend type } \\
\hline & & & & & & & & & Pro-Gly & Gly-Gly \\
\hline $\mathrm{DCC}^{*} \mathrm{~A}$ & 0.00 & -132 & 85 & 77 & 80 & -81 & -86 & -66 & V & $\mathrm{II}^{\prime}$ \\
\hline $\mathrm{DCC}^{*} \mathrm{D}$ & 0.34 & -132 & 86 & 84 & 86 & -70 & -165 & 55 & V & IV \\
\hline DACD* & 0.49 & -131 & 87 & -45 & -83 & 69 & 163 & -49 & I & IV \\
\hline $\mathrm{DCC}^{*} \mathrm{C}$ & 0.60 & -133 & 85 & 74 & 73 & -97 & -94 & 69 & V & $V^{\prime}$ \\
\hline $\mathrm{DFC}^{*} \mathrm{~A}$ & 0.66 & -132 & 86 & 156 & 84 & -67 & -69 & -50 & II & III \\
\hline $\mathrm{DCC}^{*} \mathrm{~F}$ & 0.72 & -132 & 85 & 75 & 80 & -77 & -86 & 162 & $\mathrm{~V}$ & \\
\hline $\mathrm{DFC}^{*} \mathrm{C}$ & 0.98 & -132 & 87 & 156 & 81 & -82 & -90 & 68 & II & $\mathrm{V}^{\prime}$ \\
\hline $\mathrm{DCD}^{*} \mathrm{C}$ & 1.08 & -132 & 86 & 78 & 164 & -89 & -88 & 69 & IV & IV \\
\hline DACC* & 1.09 & -131 & 88 & -21 & -81 & 80 & 86 & -76 & I & V \\
\hline $\mathrm{DFA}^{*} \mathrm{G}$ & 1.10 & -132 & 87 & 140 & 68 & 45 & -179 & -56 & II & VII \\
\hline$A^{*} F A^{*} G$ & 1.10 & 56 & 81 & 139 & 72 & 44 & -180 & -55 & II & VII \\
\hline $\mathrm{DCC}^{*} \mathrm{E}$ & 1.11 & -132 & 86 & 81 & 92 & -61 & -154 & 176 & V & \\
\hline DACA* & 1.12 & -131 & 87 & -29 & -84 & 79 & 78 & 69 & I & IV \\
\hline $\mathrm{DCA}^{*} \mathrm{C}^{*}$ & 1.14 & -133 & 82 & 76 & 59 & 74 & 84 & -71 & IV & IV \\
\hline$A^{*} C C^{*} D$ & 1.16 & 56 & 80 & 84 & 178 & -52 & -167 & 49 & IV & VII \\
\hline $\mathrm{DCC}^{*} \mathrm{G}$ & 1.16 & -132 & 87 & 82 & 88 & -65 & -159 & -64 & V & IV \\
\hline $\mathrm{A}^{*} \mathrm{FC} * \mathrm{~A}$ & 1.23 & 56 & 80 & 154 & 86 & -65 & -70 & -49 & II & III \\
\hline $\mathrm{DCG}^{*} \mathrm{C}^{*}$ & 1.27 & -132 & 87 & 75 & 167 & 68 & 86 & -75 & IV & IV \\
\hline $\mathrm{DCC}^{*} \mathrm{C}^{*}$ & 1.29 & -132 & 87 & 80 & 86 & -73 & 82 & -77 & $\mathrm{~V}$ & \\
\hline $\mathrm{DCD} * \mathrm{D}$ & 1.31 & -132 & 86 & 84 & 180 & -55 & -171 & 54 & IV & VII \\
\hline $\mathrm{DCA}^{*} \mathrm{C}^{*}$ & 1.40 & -139 & 80 & 86 & 76 & 60 & 94 & -66 & IV & III' $^{\prime}$ \\
\hline $\mathrm{A}^{*} \mathrm{CC}^{*} \mathrm{~A}$ & 1.50 & 55 & 80 & 78 & 81 & -81 & -85 & -66 & V & $\mathrm{II}^{\prime}$ \\
\hline
\end{tabular}

a All minima with $\Delta E<1.5 \mathrm{kcal} \mathrm{mol}^{-1}$.

b $E_{0}=-9.20 \mathrm{kcal} \mathrm{mol}^{-1}$.

by the ensemble composed with many stable conformations as shown in other linear oligopeptides containing Pro residue. ${ }^{12,14}$ Backbone conformations, bend types and conformational letter codes of all 22 minima with value of $\Delta E<1.5 \mathrm{kcal} \mathrm{mol}^{-1}$ are listed in Table I. The lowest-energy conformation is $\mathrm{DCC}^{*} \mathrm{~A}$ conformation with $v=0.138$ (Figure 1a), and the second lowest is $\mathrm{DCC}^{*} \mathrm{D}$ with $\Delta E=0.34$ $\mathrm{kcal} \mathrm{mol}^{-1}$ and $v=0.078$ (Figure 1b). They have common conformation DCC $^{*}$ at ValPro-Gly sequence with two hydrogenbonds (Val)CO $\cdots \mathrm{HN}(\mathrm{Gly} 3)$ and (Pro)CO .. HN(Gly4). DCC* conformation is the most favorable conformation for Val-ProGly sequence with 13 conformations in $\Delta E<$ $3 \mathrm{kcal} \mathrm{mol}^{-1^{\prime}}$ and $v=0.381$. The stable conformations of $\mathrm{Val}$ residue are restricted to only $\mathrm{D}$ and $\mathrm{A}^{*}$ conformation in $\Delta E<3$ $\mathrm{kcal} \mathrm{mol}^{-1}$ except for 11 conformations with letter code $F$ existing in $2.43 \leq \Delta E \leq 3.00$ $\mathrm{kcal} \mathrm{mol}^{-1}$. Comparing to the results for AcAla-Pro-NHMe ${ }^{12}$ Ac-Phe-Pro-NHMe, ${ }^{14}$ and Ac-Tyr-Pro-NHMe, ${ }^{14}$ stability of $\mathrm{A}^{*}$ conformation is increased. As shown in Table I, all stable conformations in $\Delta E<1.5$ $\mathrm{kcal} \mathrm{mol}^{-1}$ are $\beta$-bends at Pro-Gly portion, and also most of the stable conformations in $\Delta E<1.5 \mathrm{kcal} \mathrm{mol}^{-1}$ are $\beta$-bend at Gly-Gly portion. Bend probabilities are shown in Table II. Type II and type V, which is a distorted type II $\beta$-bend, are favorable at Pro-Gly portion. Summation of their probabilities exceeds 0.60 . These facts show that Pro-Gly sequence have a tendency to form type II $\beta$-bend. Bend probabilities at Pro-Gly portion for Ac-ProGly-NHMe, ${ }^{12}$ Ac-Pro-Gly-Gly-NHMe, and Ac-Val-Pro-Gly-Gly-NHMe show that the tendency forming bend at this portion is mainly decided by the intra- and inter-residue 


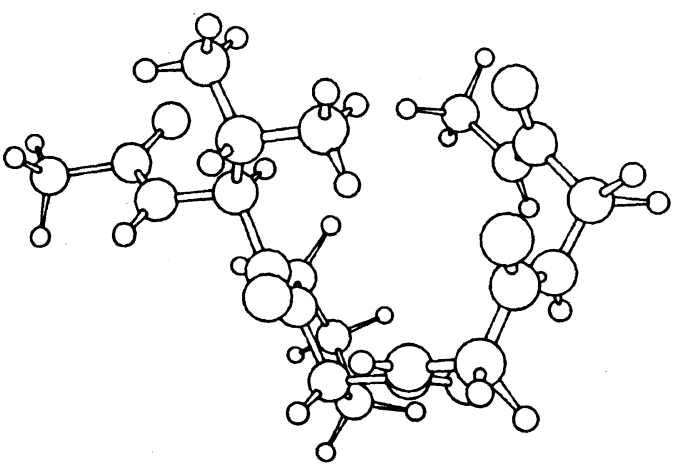

a)

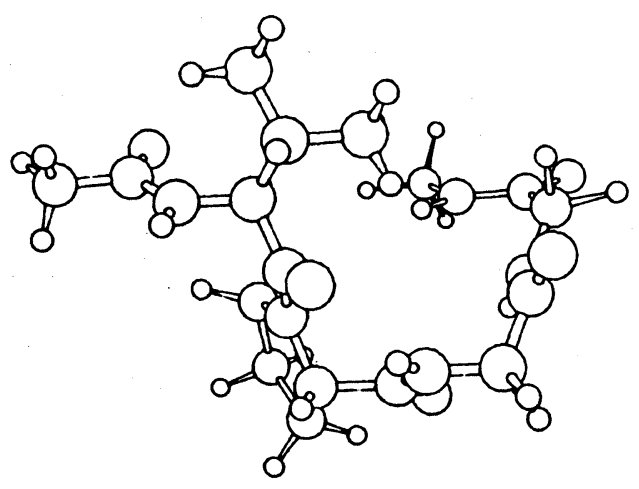

b)

Figure 1. Minimum energy conformations of Ac-ValPro-Gly-Gly-NHMe with trans Val-Pro peptide bond. a) the lowest-energy $\mathrm{DCC}^{*} \mathrm{~A}$ conformation: $\Delta E=0.00$ $\mathrm{kcal} \mathrm{mol}^{-1}$. b) the second lower-energy DCC ${ }^{*} \mathrm{D}$ conformation: $\Delta E=0.34 \mathrm{kcal} \mathrm{mol}^{-1}$.

interactions in Pro-Gly sequence, and influenced by the inter-residue interactions from Val and another Gly residue in some extents. Gly-Gly portion also represents high bend probability, but there are no favorable bend types. Bend probabilities at Gly-Gly portion for Ac-Gly-Gly-NHMe, ${ }^{15}$ Ac-Pro-Gly-Gly$\mathrm{NHMe}$, and Ac-Val-Pro-Gly-Gly-NHMe also show that the tendency to form bend at this portion is mainly governed by the intraand inter-residue interactions in Gly-Gly sequence and influenced by the inter-residue interactions from the nearest-neighbor residues (i.e., Pro residue). While they show that inter-residue interactions from the next-tonearest-neighbor residue (i.e., Val residue) are

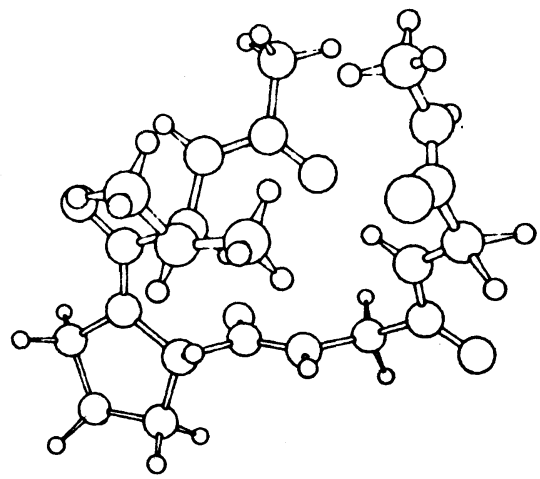

Figure 2. The lowest-energy EFD*G conformation of Ac-Val-Pro-Gly-Gly-NHMe with cis Val-Pro peptide bond.

not so important.

There are 34 low-energy minima of Ac-ValPro-Gly-Gly-NHMe with cis Val-Pro peptide bond having $\Delta E<3 \mathrm{kcal} \mathrm{mol}^{-1}$. All 8 minima with values of $\Delta E<1.5 \mathrm{kcal} \mathrm{mol}^{-1}$ are listed in Table III. The lowest-energy conformation is EFD* $\mathrm{G}$ conformation with $v=$ 0.269 (Figure 2), and the second lowest-energy conformation is EFD*A* with $\Delta E=0.39$ $\mathrm{kcal} \mathrm{mol}^{-1}$ and $v=0.140$. There are many stable conformations and no significantly favorable ones are found in this tetrapeptide system. However, EFD* conformation is the most favorable one for Val-Pro-Gly portion with $v=0.618 \quad(8$ conformations with $\Delta E<3$ $\mathrm{kcal} \mathrm{mol}^{-1}$ ) of which value is significantly larger than $v$ for the most favorable conformation for Val-Pro-Gly portion with trans peptide bond (0.318). Moreover, only EF and EA conformations were found for Val-Pro portion in the cis conformation-ensemble with $\Delta E<3 \mathrm{kcal} \mathrm{mol}^{-1}$. That is, the conformations with cis Val-Pro peptide bond are restricted to more specified conformational space than those with trans Val-Pro peptide bond (DC, DA, DF, A*F, A*C, A*A, EC, and EA were allowed for Val-Pro portion with trans peptide bond and $\Delta E<3 \mathrm{kcal} \mathrm{mol}^{-1}$ ). These results correspond to the results of conformational analysis for Ac-X-Pro-NHMe ${ }^{12,14}$ with trans or cis peptide bonds, and show that confor- 
Table II. Calculated bend probabilities of Ac-Val-Pro-Gly-Gly-NHMe

\begin{tabular}{|c|c|c|c|c|c|}
\hline \multirow{2}{*}{ Bend type and peptide } & \multicolumn{2}{|c|}{$\operatorname{trans}^{\mathrm{a}}$} & \multicolumn{3}{|c|}{$c i s^{\mathbf{a}}$} \\
\hline & Pro-Gly & Gly-Gly & Val-Pro & Pro-Gly & Gly-Gly \\
\hline I & 0.131 & 0.004 & - & 0.189 & - \\
\hline II & 0.171 & 0.005 & - & - & 0.090 \\
\hline III & 0.004 & 0.078 & - & 0.014 & - \\
\hline IV & 0.196 & 0.323 & - & 0.741 & 0.225 \\
\hline V & 0.429 & 0.032 & - & - & 0.004 \\
\hline VI & - & - & 1.000 & - & - \\
\hline VII & 0.014 & 0.105 & - & 0.044 & 0.033 \\
\hline II $^{\prime}$ & - & 0.155 & - & - & 0.094 \\
\hline III' $^{\prime}$ & - & 0.045 & - & - & 0.043 \\
\hline $\mathbf{V}^{\prime}$ & - & 0.098 & - & - & - \\
\hline Total & 0.945 & 0.844 & 1.000 & 0.988 & 0.429 \\
\hline Ac-Pro-Gly-Gly-NHMe ${ }^{b}$ & 0.889 & 0.879 & & & \\
\hline Ac-Pro-Gly-NHMe ${ }^{c}$ & 0.61 & & & & \\
\hline Ac-Gly-Gly-NHMe ${ }^{d}$ & & 0.53 & & & \\
\hline
\end{tabular}

a trans and cis peptide bonds at Val-Pro.

b Unpublished data.

c From ref 12.

${ }^{d}$ From ref 15.

Table III. Calculated minimum energy conformations ${ }^{a}$ of cis Ac-Val-Pro-Gly-Gly-NHMe

\begin{tabular}{|c|c|c|c|c|c|c|c|c|c|c|c|}
\hline \multirow{2}{*}{$\begin{array}{l}\text { Conformational } \\
\text { letter code }\end{array}$} & \multirow{2}{*}{$\frac{\Delta E_{c i s}^{\mathrm{b}}}{\mathrm{kcal} \mathrm{mol}^{-1}}$} & \multirow{2}{*}{$\phi_{\mathrm{Val}}$} & \multirow{2}{*}{$\psi_{\text {Val }}$} & \multirow{2}{*}{$\psi_{\text {Pro }}$} & \multirow{2}{*}{$\phi_{\mathrm{Gly} 3}$} & \multirow{2}{*}{$\psi_{\mathrm{Gly} 3}$} & \multirow{2}{*}{$\phi_{\mathrm{Gly} 4}$} & \multirow{2}{*}{$\psi_{\mathrm{Gly} 4}$} & \multicolumn{3}{|c|}{ Bend type } \\
\hline & & & & & & & & & Val-Pro & Pro-Gly & Gly-Gly \\
\hline EFD*G & 0.00 & -133 & 148 & 146 & 153 & -63 & -169 & -78 & VI & IV & \\
\hline EFD*A* & 0.39 & -137 & 149 & 152 & 139 & -53 & 93 & 56 & VI & IV & \\
\hline $\mathrm{EFD}^{*} \mathrm{C}$ & 0.63 & -135 & 149 & 149 & 144 & -68 & -85 & 75 & VI & IV & IV \\
\hline $\mathrm{EFC}^{*} \mathrm{D}$ & 0.63 & -141 & 150 & 155 & 69 & -91 & -143 & 32 & VI & IV & II' $^{\prime}$ \\
\hline EACD* & 0.75 & -141 & 149 & -27 & -71 & 103 & 143 & -75 & VI & I & IV \\
\hline $\mathrm{EFD}^{*} \mathrm{G}^{*}$ & 0.95 & -135 & 148 & 146 & 151 & -72 & 175 & 88 & VI & IV & \\
\hline EADA* & 1.09 & -133 & 151 & -49 & -114 & 67 & 66 & 56 & VI & I & III' $^{\prime}$ \\
\hline EAFC* & 1.40 & -139 & 149 & -41 & -75 & 159 & 103 & -72 & VI & I & II \\
\hline
\end{tabular}

a All minima with $\Delta E_{\text {cis }}<1.5 \mathrm{kcal} \mathrm{mol}^{-1}$.

b $\Delta E_{c i s}=E_{i}-E_{c i s, \min }$ and $E_{c i s, \min }=-6.14 \mathrm{kcal} \mathrm{mol}^{-1}$.

mation of Val-Pro portion are essentially decided by the intra- and inter-residue interactions within Val and Pro residues and energetically allowed conformations with cis peptide bond are more restricted than those with trans peptide bond.

$\Delta E_{\text {cis-trans, }}$. which is the energy difference between the lowest-energies of trans and cis conformations, is $2.75 \mathrm{kcal} \mathrm{mol}^{-1}$ showing that trans conformation of Ac-Val-Pro-GlyGly-NHMe is also more favorable than cis conformation as shown for proline containing oligopeptides $^{12,14}$ with intra-molecular interactions. All 34 conformations with cis Val-Pro peptide bond $\left(\Delta E<3 \mathrm{kcal} \mathrm{mol}^{-1}\right)$ form type VI $\beta$-bend at Val-Pro portion. This result corresponds to the theoretical results for $\mathrm{X}$-Pro dipeptides $^{12,14}$ and X-Pro-Y tripeptides. ${ }^{14}$ That 
is, type VI $\beta$-bend conformation is essentially favorable conformation for X-Pro (except for Gly-Pro) with cis peptide bond. Calculated bend probability for Pro-Gly portion is 0.988 and favorable bend types are IV $\left(P_{\mathrm{b}}=0.741\right)$ and I $\left(P_{\mathrm{b}}=0.189\right)$. This bend probability is higher than 0.889 for Pro-Gly portion of AcPro-Gly-Gly-NHMe with trans peptide bond and 0.945 of Pro-Gly portion of Ac-Val-ProGly-Gly-NHMe with trans peptide bond. These results show that cis conformation at Val-Pro has higher tendency to stabilize the $\beta$ bend conformation for Pro-Gly than trans conformation. $P_{\mathrm{b}}=0.429$ for Gly-Gly portion is almost the same as that of Ac-Gly-Gly$\mathrm{NHMe}^{15}\left(P_{\mathrm{b}}=0.53\right)$. That is, conformations of this portion of Ac-Val-Pro-Gly-Gly-NHMe with cis peptide bond are stabilized by intraand inter-residue interactions at Gly-Gly portion. High bend probabilities of Ac-Val-ProGly-Gly-NHMe with cis peptide bond for Val-Pro, Pro-Gly, and Gly-Gly portions characterize the favorable conformation of this tetrapeptide as triple bend conformation (11 conformations with $\Delta E<3 \mathrm{kcal} \mathrm{mol}^{-1}, v=$ $0.416)$.

\section{DISCUSSION}

Model peptides with Val-Pro-Gly-Gly sequence were synthesized and characterized their conformations in solution by Urry et al. ${ }^{6,7}$ or crystal by Ashida et al ${ }^{16,17}$ Urry et al. proposed the type II $\beta$-bend structure at ProGly portion in $t$-Boc-Val-Pro-Gly-Gly-OMe from temperature dependence of $\mathrm{NH}$ chemical shift $(\mathrm{d} \delta / \mathrm{d} T)$ in $\mathrm{CDCl}_{3}$ (with $\left.20 \% \mathrm{C}_{6} \mathrm{D}_{6}\right),{ }^{6}$ and HCO-Val-Pro-Gly-Gly-OMe by the NOE measurements in DMSO- $d_{6} \cdot{ }^{7}$ As mentioned in Results section, the calculated bend probabilities for Ac-Val-Pro-Gly-Gly-NHMe at Pro-Gly portion are 0.171 for type II and 0.429 for type $\mathrm{V}$ which is a distorted type II bend, respectively. Total bend probability of type II and type V exceed 0.60 . That is, type II and type $\mathrm{V}$ bend conformations are preferable
Table IV. Vicinal coupling constants ${ }^{3} J_{\mathrm{NH}-\mathrm{C}^{x} \mathrm{H}}$ of trans Ac-Val-Pro-Gly-Gly-NHMe

\begin{tabular}{cccc}
\hline & \multicolumn{3}{c}{${ }^{3} J_{\mathrm{NH}-\mathrm{C}^{3} \mathrm{H}}$} \\
\cline { 2 - 4 } & $\mathrm{Val}$ & $\mathrm{Gly} 3$ & $\mathrm{Gly} 4$ \\
\hline Calculated & 10.1 & 13.1 & 12.0 \\
Experimental ${ }^{\mathrm{a}}$ & 10.4 & 12.0 & 11.4 \\
\hline a $t$-Boc-Val-Pro-Gly-Gly-OMe in $\mathrm{CDCl}_{3}$ with $20 \%$ \\
$\mathrm{C}_{6} \mathrm{D}_{6}$ at $22^{\circ} \mathrm{C}$ from ref $6 . \mathrm{All}$ experimental values are \\
corrected by the relation ${ }^{3} J_{\mathrm{NH}-\mathrm{C}^{2} \mathrm{H}}=1.09 J_{\mathrm{obs}}$ in ref 13.
\end{tabular}

conformations in the ensemble of calculated stable conformations. This calculated results corresponds to the above Urry et al.'s results. ${ }^{6,7}$ Calculated and experimental ${ }^{3} J_{\mathrm{NH}-\mathrm{C}^{\alpha} \mathrm{H}}$ -in $\mathrm{CDCl}_{3}$ with $20 \% \mathrm{C}_{6} \mathrm{D}_{6}{ }^{6}$ are presented in Table IV. These results are in fairly good agreements demonstrating that calculated ensemble could represent the system of molecular conformations in non-polar solvents, even if the interactions between oligopeptides and solvents are not considered in conformational energy calculations. Moreover, NOE experiments $^{7}$ for HCO-Val-Pro-Gly-Gly-OMe in DMSO- $d_{6}$ showing that NOE of approximately $10 \%$ was observed between the $\mathrm{C}^{\alpha} \mathrm{H}$ of Pro and the NH of Gly3 support our calculated results.

Crystal structure was obtained by Ashida et al. ${ }^{16,17}$ for Boc-Val-Pro-Gly-Gly-OBzl. Backbone conformation $\left(\phi_{\mathrm{Val}}, \psi_{\mathrm{Val}}, \phi_{\mathrm{Pro}}, \psi_{\mathrm{Pro}}\right.$, $\left.\phi_{\mathrm{Gly} 3}, \psi_{\mathrm{Gly} 3}, \phi_{\mathrm{Gly} 4}, \psi_{\mathrm{Gly} 4}\right)$ of the crystal is $\left(-139^{\circ}, 158^{\circ},-62^{\circ}, 136^{\circ}, 75^{\circ}, 3^{\circ}, 74^{\circ}, 177^{\circ}\right)$ with a type II $\beta$-bend at Pro-Gly portion. Result of crystal structure corresponds to our calculated high bend probabilities of types II and $\mathrm{V}$ at Pro-Gly portion, but backbone conformations are somewhat different because of favorable hydrogen bond (NHMe)NH ... OC(Val) or (NHMe)NH ‥ OC(Gly3) which cannot be formed in Boc-Val-Pro-Gly-GlyOBzl.

Acknowledgment. We should like to thank Professor M. Sisido, Tokyo Institute of 
Technology, for presenting the program of molecular graphics.

\section{REFERENCES}

1. T. Weis-Fogh and S. O. Anderson, Nature, 227, 718 (1970).

2. W. R. Gray, L. B. Sandberg, and J. A. Foster, Nature, 246, 461 (1973).

3. J. A. Foster, E. Bruenger, W. R. Gray, and L. B. Sandberg, J. Biol. Chem., 248, 287 (1973).

4. D. W. Urry and M. M. Long, CRC Crit. Rev. Biochem., 4, 1 (1976).

5. D. W. Urry, J. Protein Chem., 3, 403 (1984).

6. M. A. Khaled, V. Renugopalakrishnan, and D. W. Urry, J. Am. Chem. Soc., 98, 7547 (1976).

7. M. A. Khaled and D. W. Urry, Biochem. Biophys. Res. Commun., 70, 485 (1970).
8. F. A. Momany, R. F. McGuire, A. W. Burgess, and H. A. Scheraga, J. Phys. Chem., 79, 2361 (1975).

9. IUPAC-IUB Commission on Biochemical Nomenclature, Biochemistry, 9, 3471 (1970).

10. M. J. D. Powell, Computer J., 7, 155 (1964).

11. S. S. Zimmerman, M. S. Pottle, G. Nemethy, and H. A. Scheraga, Macromolecules, 10, 1 (1977).

12. S. S. Zimmerman and H. A. Scheraga, Biopolymers, 16, 811 (1977).

13. V. F. Bystrov, Prog. NMR Spectroscopy, 10, 41 (19.76).

14. M. Oka, G. T. Montelione, and H. A. Scheraga, $J$. Am. Chem. Soc., 106, 7959 (1984).

15. S. S. Zimmerman and H. A. Scheraga, Biopolymers, 17, 1871 (1978).

16. H. Ayato, I. Tanaka, and T. Ashida, J. Am. Chem. Soc., 103, 5902 (1981).

17. Y. Yagi, I. Tanaka, T. Yamane, and T. Ashida, J. Am. Chem. Soc., 105, 1242 (1983). 